\title{
Intervenciones programáticas y bioeconomía: repensar la viabilidad del maguey
}

\author{
Edgar Iván Roldán $\mathrm{Cruz}^{1 \S}$ \\ Hugo Chavarría Miranda ${ }^{2}$ \\ Jessica Cano de la Rosa ${ }^{3}$
}

${ }^{1}$ Cátedra CONACyT/CEH. ${ }^{2}$ Instituto Interamericano de Cooperación para la Agricultura (hugo.chavarria@iica.int). ${ }^{3}$ Universidad Autónoma Chapingo (jessica_cano01@ outlook.com).

${ }^{\S}$ Autor para correspondencia: rcruze@gmail.com.

\section{Resumen}

El texto fundamenta la posible rentabilidad y sustentabilidad de la planta del maguey a partir del criterio biomasa y senderos de negocio, todo ello en el contexto de las disimiles lógicas de intervención, trasferencia y ciencia que se han aplicado alrededor de la planta. La revisión bibliográfica pertinente y la guía para la identificación y análisis de las posibilidades de negocios de la bioeconomía son los principales insumos necesarios para lograr tal finalidad. Así pues, es posible afirmar, pese a que el maguey aguamiel/pulque quizás desde la época colonial es la planta mayor estudiada (ligada al pasado indígena), hoy en día gravita en su última fase programática, de incidencia, sobrevivencia y preservación de saberes culturales. Más aún, el texto demuestra que aparte del pulque, es factible que el futuro del maguey abrase su multifuncionalidad y su carácter polifacético, sinergia que otorgará sin duda viabilidad de negocio al productor promedio, máxime en minimizar el factor tiempo de madurez versus desinterés por cultivar la planta.

Palabras clave: biomasa, intervenciones programáticas, maguey.

Recibido: enero de 2022

Aceptado: febrero de 2022 
Para el sistema integrado de información taxonómica (SIIT) de la Comisión Nacional para el Conocimiento y Uso de la Biodiversidad (CONABIO, 2013), la planta del maguey (de origen indígena) forma parte de la familia y género de los agaves, su distribución abarca desde el sur de los Estados Unidos de América hasta Bolivia, Paraguay, Colombia y Venezuela. México concentra cerca de 150 especies de las 200 registradas a nivel mundial, más 36 especies que pertenecen a categorías infra específicas, en suma, constituyen un total de 186 taxones (García, 2007). A pesar de que se reconocen 30 especies sobre la frontera magueyera hidalguense, la contabilidad institucional, el aporte científico y la realidad del productor, contabilizan perdida de variedades notables, grupos taxonómicos y filogenéticos (García y Galván, 1995).

Máxime a finales del Siglo XX, 1700 ha de maguey aguamiel/pulque lograban consolidar la edad propicia (más menos entre 8 y 12 años, en seis etapas transitando: medio cubo, al hilo, capón, añejo, raspa, escurrido), después de dos décadas la cuantía registra variación negativa de hasta 300; es decir, cada año desaparecen en promedio 30 ha de frontera magueyera (SIAP, 2000-2010). Tal escenario contrasta en demasía con la relevancia histórica/económica del maguey aguamiel/pulque, alguna vez considerado el eje de acumulación en la economía agraria hidalguense. Ya que en comparación con la etapa más álgida de la lógica sustitutiva de importaciones (1940-1980), se llegó a cuantificar 17 veces más a que cumplieron el período fructífero y cerca de 147 en el periodo porfirista (1876-1911), época dorada del llamado pulque fino (Loyola, 1956; DGE, 1937-1975).

La pérdida de maguey implica déficit a sin número de descriptores documentados alrededor de la planta, por mencionar algunos: conocimiento ancestral, alimentación escalonada con ciertas leguminosas y hortalizas, construcción de viviendas, sustento económico, costumbres y saberes arraigados, prácticas culturales y valor agroecológico, uso de vestimenta, bioeconomía y prototipos industriales, latifundios y haciendas pulqueras, sujetos y lucha agraria, monopolio económico y poder político, reforestación y erosión, gastronomía, alimentos y medicamentos (Ramírez, 2000).

Hoy en día la información disponible de la política pública agraria nacional reconoce al interior de la entidad hidalguense, cierta diversificación de uso de la planta del maguey: dos municipios con siembra de maguey penca (947 ha), uno maguey mixiote (23 ha) y 43 de maguey aguamiel/pulque (5 $100 \mathrm{ha}$ ), empero en cuasi todos los casos, la tendencia funcionalidad de la planta es limitada, 29 de cada 100 ha sembradas finalizan la edad propicia para su explotación (SIAP, 2018).

Por tal realidad, el texto intenta exponer los resultados de cierta vinculación académica con productores y tomadores de decisiones que permitieron fundamentar la posible rentabilidad y sustentabilidad del maguey a partir del enfoque de bioeconomía (Schmid et al., 2012), todo ello enmarcado bajo el contexto de las disimiles lógicas programáticas que ha transitado la cadena agroalimentaria (intervenciones, trasferencia y ciencia aplicada). La bioeconomía se asume como la producción y utilización intensiva en conocimientos de recursos, procesos y principios biológicos para la provisión sostenible de bienes y servicios en todos los sectores de la economía; el punto de partida es la producción sustentable de biomasa vegetal, animal y microbiana aprovechando la fotosíntesis para producir, además de alimentos, energía y amplia gama de biomateriales amigables con el ambiente (IICA, 2019).

Se busca con tal enfoque, demostrar la mayor agregación de valor posible del recurso maguey a través del uso eficiente de la biomasa tanto primaria como residual. La vinculación académica con productores y tomadores de decisiones se enmarca en el análisis y evaluación a 10 años de 
promulgarse la vigente Ley para el Manejo Sustentable del Maguey del Estado de Hidalgo, esfuerzo realizado por la Comisión de Desarrollo Agropecuario y Recursos Hidráulicos de la LXIV del Congreso Libre y Soberano del Estado de Hidalgo y miembros de la Red Científica Maguey Nopal. Tanto el criterio de biomasa y senderos de negocio, fueron los ejes rectores que se centraron en discutir y fundamentar la realidad que guarda la vigente normatividad y la incidencia programática de las políticas públicas, más aún la creación de soluciones que permitan incidir en la protección, conservación, fomento y aprovechamiento de la planta.

En el primer apartado a partir de la revisión bibliográfica pertinente, fueron esbozadas las disimiles lógicas programáticas de intervención en el maguey. Esta aproximación caracterizó a la mayoría de ellas por ciertos entramados institucionales, acciones tortuosas, rompecabezas regresivos y esfuerzos esporádicos. A partir de la evidencia histórica y actual, es posible afirmar qué: pese a que el maguey aguamiel/pulque quizás desde la época colonial es la planta mayor estudiada (ligada al pasado indígena, rural, trabajador, empobrecido y marginal), hoy en día gravita en su última fase programática de incidencia, sobrevivencia y preservación de saberes culturales. Paradójicamente ésta se caracteriza por un conjunto de acciones (lejos de una política institucional real) que buscan introducir a la lógica de la planta ciertos criterios de recuperación, reutilización y recreación.

En este apartado la representación visual del territorio es determinante, por tal a través de los Sistemas de Información Geográfica (SIG) se ejemplifica la tendencia histórica y espacial de la planta. En el SIG, el centroide representa la localidad productora del maguey, se utilizaron datos oficiales a partir de la base de datos denominada principales datos por localidad ITER, los cuales incluyen las coordenadas que permiten definir sólo los centroides de los polígonos de las localidades existentes. Obtenidos estos datos, se convirtieron dichas coordenadas al sistema UTM, datum WGS 84, ya que de origen se encuentran en formato geográfico en grados, minutos y segundos.

Una vez convertidas las coordenadas a este sistema, se representaron mediante el software ArcGis 10 y se convirtieron en archivo vectorial de puntos. A través de la clave geográfica de localidad como campo clave se unieron los datos de hectáreas productoras de maguey de casi 100 años, con el fin de representar las áreas asignadas de forma proporcional, se optó por representarlas en forma equidistante en todas direcciones, de tal forma que se representa el área asignada en forma de círculos. Para este fin, inicialmente se convirtió el área de hectáreas a metros cuadrados y se aplicó la siguiente fórmula, con el fin de obtener el radio de las áreas de influencia $r=\sqrt{\frac{\mathrm{A}}{\pi}}$. Donde: $\mathrm{r}$ es el radio que se busca representar, A es el área en metros cuadrados y $\pi$ se redondeó a 3.141597.

En el segundo apartado, a partir de la evidencia científica y la validación en la frontera magueyera hidalguense (entrevistas semi estructuradas a productores del municipio de Cardonal), se fundamenta la lógica metodológica que permitió la obtención de biomasa de una planta de maguey. Ésta se caracterizó por ser capado, variedad mediana, edad propicia 8-10 años, 145 días de explotación, mayor calidad de aguamiel, 14-15 grados Brix, de basta diversidad con respecto a las demás variedades existentes (Alfaro et al., 2017). La guía para la identificación y análisis de las posibilidades de negocios de la bioeconomía (IICA, 2019), validada por el Programa de Bioeconomía y Desarrollo Productivo del Instituto Interamericano de Cooperación de Agricultura, fue el principal insumo que permitió cuantificar tal esfuerzo metodológico. 
Los resultados a saber fueron: $601.82 \mathrm{~kg}$ penca, $370.1 \mathrm{~kg}$ aguamiel y $28.1 \mathrm{~kg}$ fibra raspado, los cuales ponderando los criterios definidos para cadenas agroalimentarias (Trigo et al., 2013), demuestran la posible viabilidad de senderos de negocio vía ingresos/ventas, más aún se reitera la vigente visión multifuncional y polifacética que aún puede dominar para la planta del maguey.

\section{Lógicas de intervención en la producción de maguey}

Primera lógica de intervención. Máxime por las históricas 100 haciendas y ranchos dedicados a la producción de maguey aguamiel/pulque, que llegaron a representar 250 mil hectáreas sobre el periodo Porfirista (Ramírez, 2000), aunado a la cercanía y conectividad ferroviaria con los tradicionales nodos industriales del país (la hoy llamada Ciudad de México y Estado de México) se figa la atención productiva por el llamado pulque fino elaborado en los Llanos de Apan, ésta es una bebida fermentada, blanca, viscosa, ácida, $\mathrm{pH} 3.5$ a 4.3 y ligeramente alcohólica: 4-7 (\%) de etanol (Ramírez, 2018). En promedio entre 1896 y en 1916 cada habitante de la capital mexicana bebía $0.9 \mathrm{~L}$ de pulque diario, la mayoría de la producción proveniente de los Llanos de Apan, esta región por la literatura coincide en delimitarla, a las fracciones pertenecientes al Estado de México, Ciudad de México, Tlaxcala, Puebla e Hidalgo, las cual circulaban alrededor del municipio de Apan (Leal y Huacuja, 1982).

Tal relevancia productiva empujó ciertos esfuerzos que intentaron intervenir, trasferir y aplicar la ciencia al maguey, empero fueron truncados por las causas/consecuencias de la reestructuración que sufrió el espacio agrícola a partir de la dotación y ejecución de tierra ejidal desde 1917 (año en que se da el primer ejercicio en el nivel estatal) y hasta 1940 (cuando finalizó la lógica agraria exportadora en México) (Roldán, 2018).

El proceso revolucionario desterró a la mayoría de la aristocracia pulquera, poder económico/político vinculado al Porfirito, responsables directos de incentivar cierta diversificación de la planta, por mencionar algunos alcances documentados: jarabe de maguey (aguamiel concentrado), agavan (néctar concentrado), alcohol industrial (farmacéutico), vinagre (fermentación de la aguamiel), goma (pegamento industrial), ixtle (fibra bastante fina, blanca y resistente) y forraje para ganado (pencas picadas y la raspadura de la misma planta) (Ramírez, 2018). Señalar además el intentó en aquella época, de minimizar lo antihigiénico en la elaboración del pulque, entre otras innovaciones: tapas de barro en lugar de piedras sobre los magueyes capados; castañas parafinadas que facilitan el lavado en su interior; bombas de extracción del aguamiel que remplazarían al uso del acocote (Olea, 2006).

Las acciones de transferencia tecnológica, algunas patentadas, en demasía fueron abaladas/realizadas por compañías privadas situadas en la hoy llamada Ciudad de México, Hidalgo y Estado de México; el ejemplo más álgido es la Compañía Expendedora de Pulques, S. C. L. y la Compañía Realizadora de Pulques, SA (Macedo, 1950). En suma, a casi 23 años del inicio de la dotación ejidal (1917), la producción hidalguense de maguey aguamiel/pulque (con limitada cuantía y escasos proceso de transferencia tecnológica) aún ocupaba el primer lugar nacional (71 mil hectáreas), $25 \%$ más grande al de su más cercano competidor (Tlaxcala) y 1.5 veces superior a la del Estado de México, entidad que registraba en aquellos años el tercer lugar nacional (Olvera et al., 2010) (Figura 1). 
Segunda lógica de intervención. Ésta se enmarca en las dos últimas décadas de la lógica sustitutiva (1960-1980), teniendo como antecedente las aceleradoras leyendas o campañas negras alrededor del maguey aguamiel/pulque: se decía que estaba fermentado con excremento de animal y ser humano. Las respuestas fueron, desde la política pública, ciertos esfuerzos institucionales que intentaron obtener la higienización, producción y trasferencia tecnológica a la planta, máxime liderados por la Comisión Nacional del Maguey (1954) y Patronato del Maguey (1960-1977), llamada después Promotora del Maguey y del Nopal (1980-1982), esfuerzos dirigidos en su mayoría por originarios de los Llanos de Apan (Herrera,1980).

Se buscaba rescatar, explotar y reformular los saberes, aportes, transferencia y valor agregado realizados durante el Porfiriato, ahora abalados en su mayoría entre otras instituciones por la Cámara Nacional de la Industria Pulquera (CNIP), Secretaría de Salubridad y Asistencia, Secretaría de la Industria, Comercio y Trabajo, Escuela de Arquitectura e Instituto de Biología (Universidad Nacional Autónoma de México), Instituto Indigenista Latinoamericano, Secretaria de Salubridad y Asistencia, Academia Mexicana de Gastronomía y Bromatología, Sociedad Mexicana de Agricultura. Tales alcances transitaron de herramientas de cambio rural (1950) a definiciones de paquetes tecnológicos (1960-1970), encausados por deficientes folletos y textos de divulgación que no permearon al productor promedio, muchas veces giraron alrededor del discurso patrimonial y moral; es decir, precaria disposición de capacidades tecnológicas asociadas a continuidad programática (Herrera,1980). En Hidalgo, pese a que la superficie sembrada de maguey aguamiel/pulque ascendía en promedio a 30000 ha en promedio (65\% de la superficie magueyera del país) y 60000 familias campesinas dependían económicamente de la planta, la industria pulquera hidalguense en términos reales disminuyó cerca de $70 \%$ (Roldán, 2015) (Figura 1).

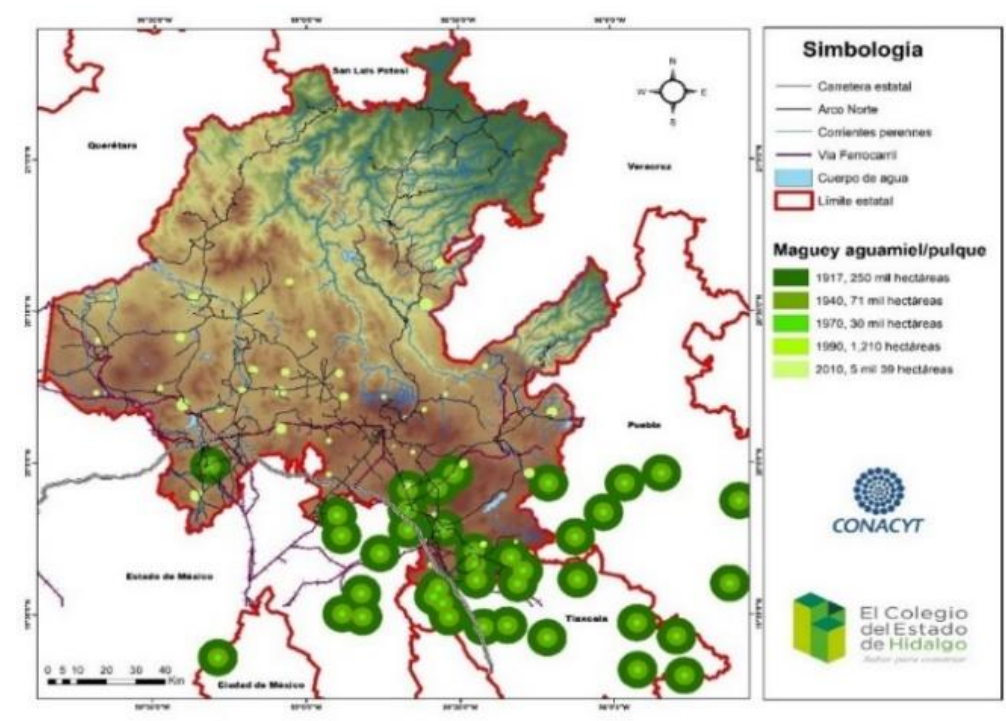

Mapa 1. Localización del maguey aguamiel/pulque (Ramírez, 2000; Olvera et al., 2010; Roldán, 2015; DGE, 1937, 1975; SIAP, 2012-2017).

Tercera lógica de intervención. Ésta se enmarca por las crisis de las políticas públicas y proyectos estratégicos alrededor de la economía abierta (1982-2000). Es decir, bajo criterios de racionalizar/encausar recursos económicos, la Promotora del Maguey y Nopal, además del 
Patronato Indígena y del Fideicomiso para el Desarrollo de la Palma y Fondo de Ejidos, se fusiona con la llamada Comisión Nacional de Zonas Áridas (CONAZA), esta fue implementada a partir de 1970 para otorgar atención a cierto conjunto importante de la población que habita en las zonas áridas y semiáridas del país (Valadez, 2014).

Así pues, se intentaron insípidas acciones por transferir subproductos de la planta o generación de valor agregado, sin éxito alguno en su mayoría. Las organizaciones y grupos de productores de maguey no pudieron organizarse e influir por el entramado e imposición de patronatos y sindicatos burocráticos que no compartían el interés de fortalecer la producción de la planta, el resultado, la desincorporación de la Cámara Nacional de la Industria Pulquera (CINP) de la llamada Cámara Nacional de la Industria de la Transformación (CANACINTRA), privatización de las centrales receptoras y del servicio ferrocarrilero asociados, al tránsito del maguey aguamiel/pulque, por ejemplo la Estación Panteco (Valadez, 2014).

En respuesta a tal escenario, la administración pública en turno divulgó sin éxito alguno en su operatividad, el Plan Regional para el Desarrollo de la Zona Magueyera (PRDZM), se argumentaba, entre otras realidades, que el rezago de la producción agrícola y el proceso inflacionario contribuyeron a mayor polarización de la riqueza y agudizado las necesidades de los estratos económicamente débiles. Sin incidencia documentada del PRDZM, la información estadística indicaba que, en promedio a finales de la última década del Siglo XX, 14 de cada 100 ha sembradas de maguey aguamiel/pulque cumplían la edad propicia (8-10 años) (SIAP, 19902000) (Figura 1).

Cuarta lógica de intervención. Esta etapa encaja a partir de la llamada alternancia política de México (2000), pues gradualmente se conjugan, lejos de la programación institucional real, serie de acciones normativas, institucionales, empíricas y científicas que paradójicamente inciden en la pronta recuperación, reutilización y recreación a la lógica productiva del maguey aguamiel/pulquero. A saber, se enlistan las tres principales de mayor incidencia.

Primera incidencia, con la publicación de la Ley de Desarrollo Agrícola Sustentable para el Estado de Hidalgo (2006) se fundamenta la Capacitación y Asistencia Técnica Rural Integral, quizás llamado extensionismo rural (POGEH, 2006). Se priorizaron desde sus primeros años, plantaciones o alimentos tradicionales de la economía agrícola hidalguense (por ejemplo, la planta del maguey) la mayoría de ellos instrumentados a través del sistema producto (SP) y Comités del Consejo Mexicano para el Desarrollo Rural Sustentable (CCMDRS) (Echeverri, 2016).

Segunda incidencia, con la promulgación de La Ley para el Manejo Sustentable del Maguey publicada el 31 de diciembre de 2011 por el Periódico Oficial del Estado (POGEH, 2011) y el respectivo reglamento divulgado el 8 de octubre de 2012 (POGEH, 2012), se intenta incidir en la propagación del maguey y polinizadores de la planta no destinada para la explotación de aguamiel/pulque, desde la reproducción asexual (hasta 2016) a la propagación in vitro (vigente). La reproducción asexual se introdujo a través de viveros comunales, instalados en casi espacios sin transcendencia histórica magueyera, bajo la justificación de que una planta adulta puede generar hasta 50 hijuelos (CIATEJ, 2017). 
Mientras que en la propagación in vitro, la germinación controlada permite obtener características deseables: precocidad, crecimiento rápido, hojas sin espinas (en bordes), más y mejor fibra (larga), mayor rendimiento de peso, bastante resistencia a sequía, humedad, enfermedades o alcalinidad, basto contenido de azúcares por mencionar algunos. Tal propagación se implementa (sin vinculación con las acciones de reproducción por hijuelo) con la edificación de viveros regionales en las principales zonas emblemáticas de mague, los servicios de consultoría son proporcionados por empresas que producen plantas a gran escala (Vázquez et al., 2011).

Tercera y última incidencia, con la publicación de la Norma199-SCFI-2017 se intenta regular y formalizar el consumo del pulque (bebida alcohólica fermentada) en dos categorías: pulque natural y pulque curado, máxime intensificar la producción pulque a mediana escala, de formar artesanal a industrial (Lappe et al., 2008). Hoy en día existen varias compañías productoras de pulque procesado con características diferentes al tradicional, en donde el principal reto de la mayoría de los esfuerzos artesanales e industriales es la adaptabilidad a las disimiles preferencias del consumidor y explotar los diversos usos del pulque/aguamiel (Aguilar et al., 2014; López et al., 2017).

\section{Biomasa y viabilidad de negocio del maguey}

A lo largo de su vida la planta, ésta puede ser aprovechada por subproductos y diversificaciones, tanto en estado entero (sin tocar), capado (castrado) y saltado (aparece otro quiote después del primero) (Vázquez et al., 2016). En el municipio del Cardonal, las actividades realizadas (productores entrevistados y explicación recabada en campo) permitieron determinar la biomasa de maguey capado. Este espacio se sitúa al noroeste del estado de Hidalgo, se distingue por su histórica marginación, clima semidesértico con temperaturas extremas, limitada precipitación y vegetación xerófila: mezquite, huizache, garambullo, nopal, biznaga, pitaya, yuca, entre otras, lo que dificulta la producción agrícola (Moreno et al., 2006). No obstante, Cardonal (llamada seí en otomí y octli en náhuatl) es el ente de mayor representatividad de maguey aguamiel/pulque en la entidad, aporta cerca de $21 \%$ del valor de la producción con rendimiento de 108 litros por hectárea, 37 de cada 100 sembradas logran con éxito la edad propicia (145 días, variedad mediana) (SIAP, 2018).

Hoy en día se reconocen 80 ha sembradas de maguey/penca, reciben en promedio $\$ 5000.00$ pesos por venta de $11 \mathrm{~kg}$ per cápita de hectárea (SIAP, 2018). Pese a la presencia próxima del Cardonal al Distrito de Riego 03 Tula, la planta del maguey corrió una suerte distinta al maíz y alfalfa, su riego fue exclusivo, además la pésima calidad de agua, para lograr su edad propicia; empero, en algunas comunidades el cultivo del maguey está en proceso de revalorización, en su mayoría por el auge ecoturístico y otras actividades propias de los adultos, además en parte por la apatía de la población joven (Vázquez, 2018).

Es posible sistematizar en seis puntos la obtención de la biomasa del maguey (capado), a saber: 1) en promedio, 145 días efectivos del ciclo productivo de una planta del maguey (variedad mediana, 8-10 años), desde la extracción/capación del meyolote hasta eliminar la planta de la melga (las hileras de esta planta). Entre primavera y otoño, periodo frecuente en la que se realiza tales operaciones; 2) el día uno de raspado de la planta, se obtiene en promedio $1.5 \mathrm{~kg}$ proveniente del meyolote, derivado de la respectiva capación; 3) para el día dos recomendable dejar reposar a la planta del maguey, no se obtienen residuos que representen biomasa; 4) en el día 3 se realiza la 
técnica de raspado, acción al día que permite obtener cerca de $200 \mathrm{~g}$ de fibra raspado (d) y $0.4 \mathrm{~L}$ de aguamiel (c); 5) día 4 al 58, continuidad en el proceso de manejo cultural de la planta, $200 \mathrm{~g}$ de fibra raspado (mexal) y en promedio 5 L de aguamiel (Ortiz et al., 2008); y 6) día 59 a 135, se obtienen los mismos $200 \mathrm{~g}$ de fibra raspado (d), empero, el rendimiento de la producción de aguamiel (c) desciende, promedio más menos 0.5 L (Muñiz et al., 2013) (Figura 2).

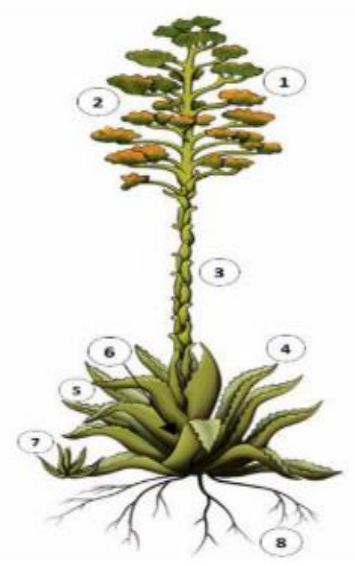

Figura 2. Morfología de la planta del maguey. 1) flor; 2) semilla; 3) quiote; 4) espina; 5) hoja o penca; 6) tallo o piña; 7) hijuelo; y 8) raíz (MNCP, 1988).

En suma, del total del ciclo productivo de aguamiel (c) de una planta de maguey (variedad mediana, 8 a 10 años), que equivale en promedio a 145 días (4.5 meses), se obtienen 286.89 L, color amarrillo cristalino, sabor dulce y olor herbáceo; constituido por agua, azucares, proteínas, gomas, sales y minerales (Lappe et al., 2008). A partir del criterio de densidad ( $\left.\mathrm{L} \mathrm{kg}^{-1}\right)$ se cuantifican $370.1 \mathrm{~kg}$ de aguamiel (c): $370.1 \mathrm{~kg}$ por $1.29 \mathrm{~g}$ litros de aguamiel. La suma parcial de fibra raspado (d) durante el ciclo productivo del aguamiel (c) registra un valor de $28.1 \mathrm{~kg} \mathrm{planta}^{-1}$.

Así pues, el planteamiento matemático de biomasa maguey (capado), es el siguiente: $\mathrm{a}=\mathrm{b}+\mathrm{c}+\mathrm{d}$. Donde: $\mathrm{a}=1000 \mathrm{~kg}$ de peso promedio de la planta del maguey (variedad mediana, 8-10 años); $\mathrm{b}=$ penca, la incógnita a definir (todo aquel material vegetativo retirado en la edad propicia); $\mathrm{c}=$ aguamiel, $370.1 \mathrm{~kg}$ y d= fibra raspado, $28.1 \mathrm{~kg}$. Sustitución y despeje: $1000=\mathrm{b}+370.1+28.1 ; 1$ $000=b+398.2 ; b+398.2=1000 ; b=1000-398.2 ; b=601.82 \mathrm{~kg}$. Asumiendo que el resto de los factores permanece constante, se indica que en promedio la biomasa efectiva para una planta del maguey capado (variedad mediana, 8-10 años, con ciclo productivo de 145 días), es de $601.82 \mathrm{~kg}$ de penca (b) (incluye hasta el mezontete), $370.1 \mathrm{~kg}$ de aguamiel (c); y $28.1 \mathrm{~kg}$ de fibra raspado (d) (incluye la piña, meyolote y metzal) (Figura 1).

Pese a que en el transcurso del tiempo se han documentado diversos aprovechamientos alrededor del maguey: 71 (MNCP, 1988) 32 (Flores et al., 2009), 78 (Vela, 2014), para fines del presente texto se exponen, a partir del criterio biomasa maguey obtenido (capado), los posibles criterios de negocios ya validados por cierta evidencia científica. Es decir, de los $601.82 \mathrm{~kg}$ de penca, se pueden obtener: Morrales de Ixtle (1 805 piezas) y penca fresca (en un día para alimentar en promedio a 20 ovinos), ambas elaboradas bajo métodos artesanales (Cuadro 1). Para el primero, la estimación de ingresos promedio por ciclo productivo de la planta, asciende a cerca de $\$ 450000.00$ pesos, mientras que, en el segundo, el ingreso/ahorro pondera cerca de $\$ 6750.00$ pesos (Cuadro 1). 
Cuadro 1. Biomasa maguey y viabilidad de negocio.

\begin{tabular}{|c|c|c|c|c|c|c|}
\hline $\begin{array}{l}\text { Criterio } \\
\text { biomasa }\end{array}$ & Rendimiento & $\begin{array}{l}\text { Justificación } \\
\text { teórica }\end{array}$ & $\begin{array}{l}\text { Método de } \\
\text { elaboración }\end{array}$ & $\begin{array}{l}\text { Estimación de } \\
\text { producción }\end{array}$ & $\begin{array}{l}\text { Ingreso } \\
\text { promedio }\end{array}$ & $\begin{array}{c}\text { Caso } \\
\text { vigente } \\
\text { mercado }\end{array}$ \\
\hline \multirow[t]{2}{*}{$\begin{array}{l}\text { Penca }= \\
601.82 \mathrm{~kg}\end{array}$} & $\begin{array}{l}1 \mathrm{~kg} \text { de penca } \\
\text { para producir } 3 \\
\text { piezas de morrales }\end{array}$ & $\begin{array}{l}\text { (Nieves et al., } \\
\text { 2007). }\end{array}$ & Artesanal & $\begin{array}{l}1805 \text { piezas } \\
\text { morrales }\end{array}$ & $\begin{array}{l}\$ 250.00 \\
\text { pesos por } \\
\text { pieza; total } \\
\$ 450000.00\end{array}$ & Hidarte \\
\hline & $\begin{array}{l}3 \mathrm{~kg} \text { de penca } \\
\text { (fresca-verde) } \\
\text { para engordar } 1 \\
\text { ovino }(20 \mathrm{~kg})\end{array}$ & (INIFAP, 2011) & Artesanal & $\begin{array}{l}\text { En un día, } 200 \\
\text { ovinos } \\
\text { alimentados por } \\
\text { penca (fresca } \\
\text { verde) }\end{array}$ & $\begin{array}{l}\text { Ahorro } \$ 6 \\
750.00 \text { pesos }\end{array}$ & $\begin{array}{l}\text { Tianguis } \\
\text { regional } \\
\text { ganado }\end{array}$ \\
\hline $\begin{array}{l}\text { Fibra } \\
\text { raspado= } \\
28.1 \mathrm{~kg}\end{array}$ & $\begin{array}{l}2100 \mathrm{~kg} \text { de fibra } \\
\text { raspado para } \\
\text { producir } 1 \text { pieza } \\
\text { papel maguey ( } 25 \\
\text { cm de alto por } 70 \\
\text { de largo, espesor } \\
\text { de } 0.21 \mathrm{ml})\end{array}$ & $\begin{array}{l}\text { (Gentry, 1982); } \\
\text { (García, 2002) }\end{array}$ & Artesanal & $\begin{array}{l}13 \text { piezas de } \\
\text { papel maguey }\end{array}$ & $\begin{array}{l}\$ 350.00 \\
\text { pesos pieza; } \\
\text { total } \$ 3 \\
145.00\end{array}$ & $\begin{array}{l}\text { Beyond } \\
\text { Gourmet }\end{array}$ \\
\hline \multirow[t]{5}{*}{$\begin{array}{l}\text { Aguamiel } \\
=370 \mathrm{~kg}\end{array}$} & $\begin{array}{l}10.36 \mathrm{~kg} \text { de } \\
\text { aguamiel para } \\
\text { producir } 1.5 \mathrm{~kg} \text { de } \\
\text { jarabe de maguey }\end{array}$ & (CIP, 2005) & Artesanal & $\begin{array}{l}45.19 \mathrm{~kg} \text { de } \\
\text { Jarabe de } \\
\text { maguey para } 180 \\
\text { frascos }(0.25 \mathrm{~g})\end{array}$ & $\begin{array}{l}\$ 100.00 \\
\text { pesos por } \\
\text { pieza; total } \\
\$ 18076.00 \\
\text { pesos }\end{array}$ & $\begin{array}{l}\text { Naturel } \\
(0.25 \mathrm{~kg})\end{array}$ \\
\hline & $\begin{array}{l}100 \mathrm{~g} \text { de aguamiel } \\
\text { para producir } 4.7 \\
\mathrm{~g} \text { de inulina }\end{array}$ & (Flores, 2008) & $\begin{array}{l}\text { Industrial, } \\
\text { secador por } \\
\text { aspersión }\end{array}$ & $\begin{array}{l}17.39 \mathrm{~kg} \text { de } \\
\text { inulina para } 35 \\
\text { presentaciones } \\
(0.5 \mathrm{~kg})\end{array}$ & $\begin{array}{l}\$ 214.00 \\
\text { pesos por } \\
\text { pieza; total } \\
\$ 7443.00\end{array}$ & $\begin{array}{l}\text { Enature } \\
(0.5 \mathrm{~kg})\end{array}$ \\
\hline & $\begin{array}{l}0.992 \mathrm{~kg} \text { de } \\
\text { pulque para } \\
\text { producir } 0.039 \mathrm{~kg} \\
\text { de etanol }\end{array}$ & $\begin{array}{l}\text { (De León et al., } \\
\text { 2008). }\end{array}$ & $\begin{array}{l}\text { Semi- } \\
\text { industrial, } \\
\text { destilación }\end{array}$ & $\begin{array}{l}27.85 \mathrm{~kg} \text { de } \\
\text { alchol etilico } \\
(70-80 \% \text { pureza }) \\
\text { para } 70 \text { botellas } \\
(0.5 \mathrm{~L})\end{array}$ & $\begin{array}{l}\$ 30.00 \text { pesos } \\
\text { por pieza; } \\
\text { total } \$ 2 \\
100.00\end{array}$ & $\begin{array}{l}\text { Protec } \\
(70 \\
\text { pureza, } \\
\text { medio } \\
\text { litro). }\end{array}$ \\
\hline & $\begin{array}{l}0.1036 \mathrm{~kg} \text { de } \\
\text { aguamiel para } \\
\text { producir } 0.508 \mathrm{~kg} \\
\text { de goma maguey }\end{array}$ & $\begin{array}{l}\text { (Lappe et al., } \\
\text { 2008). }\end{array}$ & $\begin{array}{l}\text { Semi- } \\
\text { industrial, } \\
\text { evaporización } \\
\text { y el secado }\end{array}$ & $\begin{array}{l}1814.28 \mathrm{~kg} \mathrm{de} \\
\text { goma maguey } \\
\text { para } 8 \text { frascos } \\
(0.25 \mathrm{~g})\end{array}$ & $\begin{array}{l}140 \text { pesos } \\
\text { por pieza; } \\
\text { Total, } 1.015\end{array}$ & $\begin{array}{l}\text { Mi } \\
\text { granero } \\
(0.25 \mathrm{~g}) .\end{array}$ \\
\hline & $\begin{array}{l}5.18 \mathrm{~kg} \text { de } \\
\text { aguamiel por } 9.92 \\
\mathrm{~kg} \text { de pulque }\end{array}$ & $\begin{array}{l}\text { (Aguilar et al., } \\
\text { 2014). }\end{array}$ & $\begin{array}{l}\text { Industrial, } \\
\text { doble } \\
\text { pasteurización } \\
\text { y enlatado }\end{array}$ & $\begin{array}{l}71428 \mathrm{~L} \text { de } \\
\text { pulque para } 2 \\
138 \text { de pulque } \\
\text { enlatado }(0.334 \\
\text { L) }\end{array}$ & $\begin{array}{l}\$ 20.00 \text { pesos } \\
\text { por pieza; } \\
\text { total } \$ 42 \\
771.00\end{array}$ & $\begin{array}{l}\text { Pulque } \\
\text { néctar } \\
\text { del razo } \\
(0.334 \mathrm{~L})\end{array}$ \\
\hline
\end{tabular}

Para los $380 \mathrm{~kg}$ de aguamiel conseguidos, los subproductos y diversificaciones que pueden obtenerse elaborados desde métodos artesanales hasta industriales contabilizan ingresos promedio de, por orden de intensidad, \$42 771.00 pesos (pulque enlatado); 18076.00 pesos (jarabe de 
maguey); \$7 443.00 pesos (inulina); \$2 100.00 pesos (alcohol etílico) y \$1 015.00 pesos (goma de maguey) (Cuadro 1). Finalmente, para los $28.1 \mathrm{~kg}$ de fibra raspado, con 13 piezas del papel maguey se recaudan (explotación artesanal) cerca de \$3 145.00 pesos en promedio (Cuadro 1).

Importante indicar antes de finalizar el apartado, si bien las condiciones de edáficas y climáticas influyen en el crecimiento de la planta, también es importante tanto las labores/estrategias de los productores como las respuestas de la política pública agrícola a saber, se exponen tres condiciones a ponderar. Primera condición: la plantación escalonada (validada), después de cuatro años de crecimiento de la planta del maguey vía vivero $(50$ a $70 \mathrm{~cm})$, se trasplanta a un sistema de alta densidad, donde se planifica su crecimiento a cuatro años más, con base en una reconversión productiva gradual y escalada, uso eficiente e intensivo del espacio.

En una hilera se trasplantan los magueyes provenientes del vivero, de cuatro años de crecimiento, con seis metros de separación entre cada fila y dos metros entre cada planta. Pasados dos años se vuelve a sembrar otra hilera de magueyes provenientes de vivero, ahora con una separación de dos metros entre una de las hileras de las plantas de seis años de crecimiento, cuatro años en vivero y dos en campo y a cuatro metros de la otra hilera. Después de cuatro años, se siembra nuevamente una hilera de magueyes provenientes de vivero, con una separación entre cada fila de $2 \mathrm{~m}$. Teniendo así, tres hileras de crecimiento escalonado: ocho años, seis años y cuatro años (González, 2017).

Segunda condición: las históricas prácticas culturales (podas, deshierbe, fertilización, daño cuticular, al menos dos veces al año) y el manejo de plagas/enfermedades (el llamado picudo, la principal plaga que afecta a la planta casi todo el año, provoca la degradación de los tejidos y la muerte de la planta, desde 30 hasta 93\%), las cuales se conjunta con la elaboración de biopreparados artesanales, por mencionar algunos: bioestimulantes, enraizadores, biofungicidas, bioinsecticidas, biorepelentes, biofertilizantes (Solís et al., 2001; Vázquez et al., 2016).

Y finalmente la tercera condición: derivado de la distribución amplia en ambientes limitantes (suelos someros, infértiles y secos), es determinante el análisis de la variación genética del maguey que permita alcanzar la conservación y la realización de programas de mejoramiento genético. Los estimadores de la extensión de la variación genética disponible, contribuye al monitoreo de germoplasma y permite predecir ganancias genéticas potenciales (Moreno y Cubero, 1993).

\section{Conclusiones}

Se demuestra la posible viabilidad de sustituir la lógica dominante de tirar la planta después de cada sistema de explotación del maguey (penca, mixiote, aguamiel), por una modelo circular (trazabilidad) en la que todos los residuos se puedan usar y transformar a recursos tangibles (biomasa). La planta del maguey puede ser nuevamente el recurso de mayor rentabilidad dada la gran cantidad de productos que se pueden obtener y de la creciente demanda que cada uno de ellos tiene (Narváez et al., 2016). Hay que decir que la ciencia debe seguir trabajando en el nivel de conocimiento de la diversidad genética del maguey aguamiel/pulque; ya que, en comparación con otros géneros y especies, existen limitaciones, la mayoría provienen de caracteres morfológicos y citológicos (Gil et al., 2001). 


\section{Literatura citada}

Alfaro, R. G.; Legaria, S. J. y Rodríguez, P. J. 2017. Diversidad genética en poblaciones de agaves pulqueros (Agave spp.) del noriente del Estado de México. Rev. Fitotec. Mex. 30(1):1-12.

Aguilar, J. B.; Enríquez, D. J.; Rodríguez, O. G; Granados, S. D. y Martínez, C. B. 2014. El estado actual de Agave salmiana y A. mapisaga del Valle de México. México. Rev. Mex. Agroec. 1(2):106-120.

CIATEJ. 2017. Centro de Investigación y Asistencia en Tecnología y Diseño del Estado de Jalisco A. C. Panorama del aprovechamiento de los Agaves en México. Centro de Investigación y Asistencia en Tecnología y Diseño del Estado de Jalisco AC. Guadalajara, Jalisco, México. $303 \mathrm{p}$.

CIP. 2005. Centro Internacional de la Papa. Jarabe de yacón: principios y procesamiento. CIAP. Lima, Perú. 32 p.

CONABIO. 2013. Comisión Nacional para el Conocimiento y Uso de la Biodiversidad. Sistema Integrado de Información Taxonómica (SIIT). https://www.biodiversidad.gob.mx /especies/CAT.

De León, A. E.; Jiménez, P. M.; Ordoñez, L.; Flores, J. and Barba, A. 2008. Characterization of volatile compounds from ethnic Agave alcoholic beverages by gas chromatography-mass spectrometry. Food Technol. 46(4):448-455.

DGE. 1937. Dirección General de Estadística. Primer censo agrícola ganadero. Secretaría de la Economía Social. Gobierno de la República. México. 75 p.

DGE. 1975. Dirección General de Estadística. V Censo agrícola ganadero y ejidal. Dirección General de Estadística. México. 99 p.

Echeverri, P. R. 2016. Estrategia nuevo extensionismo en México. SAGARPA, INCA Rural, IICA, Red para la Gestión Territorial del Desarrollo Rural, RED GTD-IICA. San José Costa Rica. $63 \mathrm{p}$.

Flores, M. A. 2008. Rescate del maguey pulquero (Agave salmiana otto ex salm) en el estado de Tlaxcala y Puebla. México. Instituto Tecnológico del Altiplano Potosino. 70 p.

Flores, M. A.; Castañeda, H. E.; Sánchez, P. F y Ruíz, L. J.2009. Mecanismos de conservación y uso del maguey pulquero Agave salmiana en el Altiplano Mexicano. Sociedad Mexicana de Agricultura Sostenible AC. México. 20 p.

García, A. J. 2007. Los agaves de México. México. Rev. Cienc. 87(1):14-23.

García, M. A. 2002. Distribution of the fenus Agave (Agavaceae) and its endemic species in Mexico. US. Cactus and Succulent J. 74(1):177-187.

García, M. A. y Galván, V. R. 1995. Riquezas de las familias Agaváceae y Nolinaceae en México. México. Boletín de la Sociedad Botánica de México. 56(1):7-24.

González, M. J. 2017. Pulque, la bebida de los dioses al alcance de todos corporativo magueyeros San Isidro SA de CV. In: innovar para competir 40 casos de éxito. Secretaría de Agricultura, Ganadería, Desarrollo Rural- Instituto Interamericano de Cooperación para la AgriculturaCoordinadora Nacional de las Fundaciones Produce, AC. DF, México. 99-102 pp.

Gentry, H. S. 1982. Agaves of Continental North America. University of Arizona Press. USA. $670 \mathrm{p}$.

Gil, V. K.; Chavira, M. G.; De la Vega, M. O.; Simpson, J. and Vandemark, G. 2001. Analysis of genetic diversity in Agave tequilana var. Azul using RAPD markers. Euphytica. 119(1):335-341. 
Herrera, C. A. 1980. Política y gobierno en Hidalgo. Fundación Arturo Herrera Cabañas. Pachuca, Hidalgo. 247 p.

IICA. 2019. Instituto Interamericano de Cooperación para la Agricultura. Guía para la identificación y análisis de las posibilidades de negocios de la Bioeconomía. Costa Rica. $200 \mathrm{p}$.

INIFAP. 2011. Instituto Nacional de Investigaciones Forestales, Agrícolas y Pecuarias. Alimentación para ovinos de la región norponiente de Tlaxcala. (Comp.) Galaviz, R. J. R.; Zaragoza, R. J. L. y Corona, J. V. Tlaxcala, México. 32 p.

Lappe, O. P; Moreno, T. R.; Arrizon, G. J.; Herrera, S. T.; García, M. A. and Gschaedler, M. A. 2008. Yeasts associated with the production of Mexican alcoholic non-distilled and distilled Agave beverages. Inglaterra. FEMS Yeast Research. 8(7):1037-1052.

Leal, J. F. y Huacuja, R. M. 1982. Economía y sistema de haciendas en México. La hacienda pulquera en el cambio. Siglos XVIII, XIX y XX. (Ed.) Era. DF, México. 200 p.

López, M. C. A.; García, G. M. y Quintero, R. C. 2017. Biotecnología Alimentaria. (Ed.) Limusa Noriega. DF, México. 636 p.

Loyola, M. E. 1956. La industria del pulque. Banco de México-Departamento de Investigaciones Industriales. DF, México. 348 p.

Macedo, M. E. 1950. Manual del magueyero. (Ed.) Agrícolas Trucco. DF, México. 165 p.

Moreno, B.; Garret, R. M. G. y Fierro, A. U. J. 2006. Otomíes del Valle del Mezquital. CDI. DF México. 54 p.

Moreno, G. J. y Cubero, J. I. 1993. Selection strategies and choice of breeding materials. In: plant breeding: principles and prospects. (Ed.). Hayward, M. D.; Bosemark, N. O.; Romagosa, Chapman \& Hall. London Reino Unido. 281-313 pp.

Muñiz, M. D.; Rodríguez, J. R.; Rodríguez, H. R.; Contreras, E. J. y Aguilar, G. C. 2013. Producción artesanal del aguamiel: una bebida tradicional mexicana. México. Acta Química Mexicana. 10(5):12-19.

MNCP. 1988. Museo Nacional de Culturas Populares. El maguey, árbol de las maravillas. Robles Hermanos y Asociación. DF, México. 200 p.

Narváez, S. A.; Martínez, S. T. y Jiménez, V. M. 2016. El cultivo de maguey pulquero: opción para el desarrollo de comunidades rurales del altiplano mexicano. México. Rev. de Geografía Agrícola. 56(1):33-44.

Nieves, H. G.; Héctor, S. L. y Ayala, R. J. 2007. Fibras y artesanías con agave en Jalisco. In Agaves del Occidente de México. (Ed.). Vázquez, G. A.; Hernández, V. M.; Flores, B. G. y Vargas, R. V. Universidad de Guadalajara, Centro de Investigación y Asistencia en Tecnología y Diseño del Estado de Jalisco, Consejo Regulador del Tequila, Lousiana State University. México. 123-133 pp.

Olea, F. A. 2006. Memoria sobre el maguey mexicano y sus diversos productos. Compendio de Geografía de México. Consejo Nacional para la Cultura y las Artes. DF, México. 269 p.

Olvera, L. J.; Romero, O. y Jiménez, G. 2010. El ocaso de un cacicazgo: transformaciones agrarias e industriales en los Llanos de Apan, Hidalgo. El Colegio de Tlaxcala, AC. DF, México. 187 p.

Ortiz, B. R.; Pourcelly, G.; Doco, T.; Villiams, P.; Dormer, M. and Belleville, M. P. 2008. Analysis of the main components of the aguamiel produced by the magueypulquero (Agave mapisaga) throughout the harvest period. J. Agric. Food Chem. 56(1):3682-3687.

POGEH. 2006. Ley de Desarrollo Agrícola Sustentable para el Estado de Hidalgo. Periódico oficial del Gobierno del Estado de Hidalgo. 
POGEH. 2011. La ley para el manejo sustentable del maguey. Periódico oficial del Gobierno del Estado de Hidalgo. 28 p.

POGEH. 2012. Reglamento de La Ley para el manejo sustentable del maguey. Periódico oficial del Gobierno del Estado Hidalgo. Gobierno del Estado de Hidalgo. 29 p.

Ramírez, R. M. 2000. Ignacio Torres Adalid y la industria pulquera. Plaza y Valdés. DF, México. $347 \mathrm{p}$.

Ramírez, R. R. 2018. La querella por el pulque: auge y ocaso de una industria mexicana, 18901930. El Colegio de Michoacán. Zamora, Michoacán. 580 p.

Roldán, C. E. 2015. Organización económica y desarrollo regional del estado de Hidalgo: pasado y presente. El Colegio del Estado de Hidalgo. Pachuca, Hidalgo. 305 p.

Roldán, C. E. 2018. Reparto agrario. Reestructuración del espacio y producción alimentaria en Hidalgo México, 1917-1940. México. Rev. de El Colegio de San Luis Nueva época. 16(1):229-263.

Schmid, O.; Padel, S. and Levidow, L. 2012. The bio-economy concept and knowledge base in a public goods and farmer perspective. Bio-based and Applied Economics. 1(1):47-63.

SIAP. 1990. Servicio de Información Agroalimentaria y Pesquera. Producción anual agrícola 2000. https://nube.siap.gob.mx/cierreagricola/.

SIAP. 2000. Servicio de Información Agroalimentaria y Pesquera. Producción anual agrícola 2000. https://nube.siap.gob.mx/cierreagricola.

SIAP. 2010. Servicio de Información Agroalimentaria y Pesquera. Producción anual agrícola 2010. https://nube.siap.gob.mx/cierreagricola/.

SIAP. 2012. Servicio de Información Agroalimentaria y Pesquera. Producción anual agrícola 2010. https://nube.siap.gob.mx/cierreagricola/.

SIAP. 2017. Servicio de Información Agroalimentaria y Pesquera. Producción anual agrícola 2010. https://nube.siap.gob.mx/cierreagricola/.

SIAP. 2018. Servicio de Información Agroalimentaria y Pesquera. Producción anual agrícola 2010. https://nube.siap.gob.mx/cierreagricola/.

Solís, A. J.; González, H. H.; Leyva, V. J.; Equihua, A. M.; Flores, M. F. y Martínez, A. G. 2001. Scyphophorus acupunctatus Gyllenhal, plaga del agave tequilero en Jalisco, México. Agrociencia. 35(6):663-670.

Trigo, J. E.; Henry, G.; Sanders, J.; Schurr, U.; Ingelbrecht, I.; Revel, C. and Pedro, R. 2013.Towards bioeconomy development in Latin America and the Caribbean. In: Jaramillo, E. (Ed.). Towards a Latin America and Caribbean knowledge-based bioeconomy in partnership with Europ. 15-41 pp.

Valadez, M. M. 2014. Pulque limpio/pulque sucio: disputas en torno a la legitimidad y la producción social del valor. Colombia. Rev. Colomb. Antropol. 50(2):41-63.

Vázquez, G. A.; Alipant, F. M. M.; Estrella, C. N. G.; Ortiz, T. E.; Ramírez, J. J. y Ramírez, A. M. 2016. El maguey pulquero: una planta multifuncional y polifacética: los usos desde una visión mestiza e indígena. Buenos Aires, Argentina. Scripta Ethnologica. 38(1):65-87.

Vázquez, G. A. 2018. Usos, prácticas culturales y capital natural del maguey pulquero (Agave salmiana) en dos grupos étnicos, en el territorio del Altiplano Central mexicano. Colegio de Posgraduados en Ciencias Agrícolas. Tesis de Doctorado en Ciencias. 165 p.

Vázquez, D. E.; García-Nava, R.; Peña-Valdivia, C.; Ramírez-Tobías, H. y Morales-Ramos, V. 2011. Tamaño de la semilla, emergencia y desarrollo de la plántula de maguey (Agave salmiana Otto ex Salm-Dyck). Rev. Fitotec. Mex. 34(3):161-173.

Vela, E. 2014. Los usos de los magueyes. México. Arqueología Mexicana. 57(1):55-58. 